\title{
Editorial Note: Experimental Observation of Optical Rotation Generated \\ in Vacuum by a Magnetic Field \\ [Phys. Rev. Lett. 96, 110406 (2006)]
}

E. Zavattini, G. Zavattini, G. Ruoso, E. Polacco, E. Milotti, M. Karuza, U. Gastaldi, G. Di Domenico, F. Della Valle,

R. Cimino, S. Carusotto, G. Cantatore, and M. Bregant

(PVLAS Collaboration)

(Received 7 August 2007; published 20 September 2007)

DOI: 10.1103/PhysRevLett.99.129901

PACS numbers: $12.20 . \mathrm{Fv}, 07.60 . \mathrm{Fs}, 14.80 . \mathrm{Mz}, 99.10 . \mathrm{Np}$

The observed vacuum optical rotation signal reported in [1] has now been excluded by more recent results from the PVLAS Collaboration [2], which show that it was due to an instrumental artifact and was not of physical origin. These new data therefore also exclude the possible interpretation of the signal reported in [1], as caused by the existence of a light, neutral, spin-zero particle.

[1] E. Zavattini et al., Phys. Rev. Lett. 96, 110406 (2006).

[2] E. Zavattini et al., arXiv:0706.3419. 